\title{
Enhanced downregulation of transforming growth factor- $\beta 2$ in rat retinal pigment epithelium cells by adeno-associated virus-mediated ribonucleic acid interference combined with ultrasound or microbubbles
}

\author{
HONGLI LI ${ }^{1}$, CAIFENG WAN ${ }^{1}$, LIANFANG DU ${ }^{2}$ and FENGHUA LI ${ }^{1}$ \\ ${ }^{1}$ Department of Ultrasound, Ren Ji Hospital, School of Medicine, Shanghai Jiao Tong University, Shanghai 200127; \\ ${ }^{2}$ Department of Ultrasound, Shanghai First People's Hospital, Shanghai 200080, P.R. China
}

Received March 23, 2014; Accepted October 6, 2014

DOI: $10.3892 / \mathrm{mmr} .2014 .2845$

\begin{abstract}
The present study was designed to determine the efficiency and safety of ultrasound (US) and/or US contrast agent microbubbles (MBs) in the delivery of type 2 recombinant adeno-associated virus-delivered transforming growth factor- $\beta 2$ short hairpin ribonucleic acid encoding the enhanced green fluorescent protein gene (rAAV2-TGF 32 shRNA-EGFP) and the downregulation of TGF $\beta 2$ in rat retinal pigment epithelium (RPE-J) cells. The effects of US and/or MBs on the delivery of rAAV2-EGFP and rAAV2-TGFß2 shRNA-EGFP were evaluated by fluorescence microscopy and flow cytometry. The potential toxicity of cell viability under various US or MB conditions was assessed by CellTiter $96^{\circledR}$ AQueous One solution cell proliferation assay. The level of TGF $\beta 2 \mathrm{mRNA}$ in RPE-J cells under various conditions was estimated by reverse transcription-quantitative polymerase chain reaction analysis. The results obtained demonstrated that low-intensity US $\left(0.5 \mathrm{~W} / \mathrm{cm}^{2}\right.$ and $\left.30 \mathrm{sec}\right)$ or SonoVue (MB:cell ratio, 40:1) increased the delivery efficiency of rAAV2-EGFP and rAAV2-TGFß2 shRNA-EGFP to RPE-J cells, whereas the combination of US with MBs did not further increase but instead decreased rAAV transfection. Under the optimal conditions of rAAV delivery, enhanced TGF 32 gene silencing with a combination of US or SonoVue with rAAV2-TGFß2 shRNA resulted in a significant decrease in mRNA levels compared with rAAV2-TGFß2 shRNA alone. US or SonoVue was used safely to enhance the delivery of rAAV2-TGF 32 shRNA to RPE-J cells. A combination of the biological (rAAV2-TGF $\beta 2$
\end{abstract}

Correspondence to: Professor Fenghua Li, Department of Ultrasound, Ren Ji Hospital, School of Medicine, Shanghai Jiao Tong University, 1630 Dongfang Road, Shanghai 200127, P.R. China E-mail: fenghualipro@163.com

Key words: ultrasound, microbubbles, recombinant adeno-associated virus, RNA interference, retinal pigment epithelium cells
shRNA) and physical (US or SonoVue) approaches downregulated the mRNA level of TGF $\beta 2$ more effectively.

\section{Introduction}

Proliferative vitreoretinopathy (PVR) is characterized by the formation of contractile membranes in the vitreal cavity and on both surfaces of the retina. Retinal pigment epithelial (RPE) cells are considered to play a pivotal role in the formation and the contraction of these membranes, and are known to secrete a wide range of growth factors which are significant in the progression of the disease, including transforming growth factor- $\beta$ (TGF $\beta$ ), platelet-derived growth factor and fibroblast growth factor (1). TGF $\beta 2$ is considered to be the predominant TGF $\beta$ isoform in the posterior segment. TGF $\beta 2$ has been identified in membranes excised from patients with PVR by immunohistochemistry. TGF 32 concentration is elevated in the vitreous of patients with the disease $(2,3)$. Therefore, targeting of the TGF $\beta 2$ gene with a small interfering RNA (siRNA) in RPE cells has the potential to prevent the development of PVR.

RNA interference (RNAi) is a promising biological strategy for the treatment of diverse diseases; however, the therapeutic application of siRNA has been limited by its instability and poor cellular uptake efficiency (4). If the siRNA in vivo is to become effective with high efficiency and stability in the long-term, a vector is required. Currently, the virus vector is the main vector applied in RNAi research. Recombinant adeno-associated virus (rAAV), characterized by low immunogenicity and long exogenous gene expression time, is particularly applicable to the gene therapy of ophthalmology. The identification of the rAAV vector of RNAi provides the premise for the enhanced use of RNAi technology to silence related genes in the treatment of ophthalmic diseases $(5,6)$. However, the solution to the low infection rate of rAAV has not yet been elucidated $(7,8)$. The use of ultrasound (US) and microbubbles (MBs) has been proven to be safe and efficient in various organs. Our previous studies demonstrated that US with MBs enhanced rAAV transfection efficiency in ARPE19 cells $(9,10)$. The present study was designed to determine whether US and/or MBs not only facilitate the delivery of rAAV2-enhanced green fluorescent protein (EGFP) but also 
the downregulation of TGF 32 combined with rAAV2-TGF $\beta 2$ shRNA in rat RPE cells. To the best of our knowledge, only a few studies have been published with regard to US/MBs combined with AAV-mediated RNAi in the sphere of ophthalmology. If successful, such an approach may provide an alternative gene delivery method for RNAi in RPE cell-related diseases.

\section{Materials and methods}

rAAV2-EGFP-based transfection in RPE-J cells with US and/or US contrast agent

Cell culture. A rat RPE-J cell line was obtained from the American Type Culture Collection (CRL-2240; ATCC, Rockville, MD, USA). The cells were cultured to $70-80 \%$ confluence in Dulbecco's modified Eagle's medium (Gibco, Grand Island, NY, USA) supplemented with $4 \%$ fetal bovine serum at $37^{\circ} \mathrm{C}$, in $5 \% \mathrm{CO}_{2} / 95 \%$ air. RPE cells were placed onto every other well of a 24 -well plate at a concentration of $1 \times 10^{5}$ cells per well prior to infection.

US contrast agent and virus. SonoVue, a lipid-shelled US contrast agent filled with sulfur hexafluoride gas, composed of $\sim 2 \times 10^{8}$ per $\mathrm{ml} \mathrm{MBs}$, and having an average diameter of 2.5-6.0 mm, was purchased from Bracco (Milan, Italy). Perfluoropropane-albumin microspheres, an albumin-shelled US contrast agent filled with perfluoropropane gas, composed of $\sim 5 \times 10^{8}$ per $\mathrm{ml} \mathrm{MBs}$, and having an average diameter of 3-4.5 $\mu \mathrm{m}$, was purchased from Jing Da pharmaceutical Co., Ltd., Hu Nan, China; approval number S20083098). Finally, rAAV2-EGFP $\left[2 \times 10^{12}\right.$ vector genomes per milliliter $\left.(\mathrm{vg} / \mathrm{ml})\right]$ was purchased from Vector Gene Technology Company Ltd. (Beijing, China). A dose of $1 \times 10^{4}$ MOI was used (multiplicity of infection: vector genomes/number of cells).

US exposure protocol. The volume per well was $150 \mu \mathrm{l}$ when exposed to US and the method of transfection configuration was the same as we previously reported (9). A therapeutic US device (Topteam 161, Chattanooga Medical Supply, Inc., Chattanooga, TN, USA) was used as previously described (9). We set the experimental conditions as follows: frequency, 1 $\mathrm{MHz}$; US intensity, 0.5 and $1 \mathrm{~W} / \mathrm{cm}^{2}$; duration, 30, 60 and $90 \mathrm{sec}$; pulse wave with $50 \%$ duty cycle; with or without MBs (SonoVue or perfluoropropane-albumin microspheres; MB:cell ratio, 20:1, 40:1 and 60:1). RPE cells were infected by rAAV alone (control) or in combination with US (rAAV+US), SonoVue (rAAV+SonoVue), perfluoropropane-albumin microspheres (rAAV+microsphere) or US-targeted microbubble destruction (UTMD; rAAV+US+SonoVue and rAAV+US+microsphere group; MB:cell ratio, 20:1).

Gene transfer efficiency. Forty-eight hours after rAAV transfection, EGFP expression was observed and photographed using an inverted fluorescence microscopy (Zeiss Axiovert S100, Carl Zeiss, Oberkochen, Germany). The ratio of infected cells was examined by a fluorescence-activated cell sorter (FACS; Epics XL, Beckman Coulter, Miami, FL, USA).

Cell damage assay. RPE cells were placed into groups of four adjacent holes of a 96-well plate (with every other four adjacent holes left empty), at a concentration of $8 \times 10^{4}$ cells per well. The cells were exposed to US (frequency, $1 \mathrm{MHz}$; US intensity, 0.5 and $1 \mathrm{~W} / \mathrm{cm}^{2}$; duration, 30, 60 and $90 \mathrm{sec}$; $50 \%$ duty cycle) or SonoVue (MB:cell ratio, 20:1, 40:1, 60:1 and 80:1). The volume per well was $100 \mu 1$. Twenty-four hours after treatment and under various exposure parameters, MTS assay (CellTiter $96^{\circledR}$ AQueous One solution cell proliferation assay, Promega, Madison, WI, USA) was performed to obtain the absorbance value. The absorbance value of RPE-J cells that were not treated was used as a control.

rAAV2-TGFß2 shRNA-U6-EGFP-based transfection in RPE-J cells with US or SonoVue

Design and screening of siRNAs in RPE-J cells. The siRNA targeting the rat TGF $\beta 2$ (gene ID 81809) coding sequence (three pairs, Table I) and control siRNA were designed and synthesized by Shanghai GenePharma Co., Ltd. (Shanghai, China). To avoid possible off-target effects in the rat, these target sequences had been used to screen Gen-Bank using BLAST. To test siRNAs, RPE-J cells were co-transfected with synthetic siRNAs and Lipofectamine 2000 (Invitrogen, Carlsbad, CA, USA). Finally, the optimal RNAi target sequence (TGFß2-771) was selected by the expression level of mRNA.

rAAV2-TGF 32 shRNA-U6-EGFP constructs. rAAV2-TGF 32 shRNA-U6-EGFP was constructed by Vector Gene Technology Company Ltd. on the basis of the TGF $\beta 2-771$ we selected. Transgenes were driven by the U6 promoter. The titer of genome particles was $2.7 \times 10^{11} \mathrm{vg} / \mathrm{ml}$. rAAV2-neg-shRNA-U6-EGFP was also constructed by the same company. The titer of genome particles was $4.4 \times 10^{11} \mathrm{vg} / \mathrm{ml}$.

US- or SonoVue-mediated rAAV2-TGF 32 shRNA-EGFP transfected RPE cells. RPE cells were placed into a 12-well plate at a concentration of $1 \times 10^{5}$ cells per well. The method of transfection configuration was the same as described above. The dose of rAAV2-TGF 32 shRNA-EGFP and rAAV2-negshRNA-EGFP was $5 \times 10^{4} \mathrm{MOI}$.

FACS-examined EGFP expression. rAAV2-TGF $\beta 2$ shRNA-EGFP was transfected into RPE cells with US $\left(0.5 \mathrm{~W} / \mathrm{cm}^{2}\right.$ and $\left.30 \mathrm{sec}\right)$ or SonoVue (MB:cell ratio, 40:1). rAAV2-TGFß2 shRNA-EGFP transfected alone served as the control. Forty-eight hours after transfection, EGFP expression (ratio of positive cells and mean fluorescence density) was examined by FACS.

Reverse transcription-quantitative polymerase chain reaction $(R T-q P C R)$ analysis of TGF $\beta 2$ gene expression. The level of TGF $\beta 2$ mRNA in RPE-J cells was estimated by RT-qPCR analysis $48 \mathrm{~h}$ after rAAV2-TGF 32 shRNA-EGFP was transfected with US $\left(0.5 \mathrm{~W} / \mathrm{cm}^{2}\right.$ and $\left.30 \mathrm{sec}\right)$ or SonoVue (MBs:cells, 40:1). RPE-J cells that were not treated were used as a control. To determine the efficiency of gene delivery by a combination of US or SonoVue, the cells transfected with rAAV2-neg-shRNA-EGFP were also collected to measure the mRNA levels $48 \mathrm{~h}$ post-transfection. Total RNA was harvested using a TRIzol reagent kit (Invitrogen, Karlsruhe, Germany) and then reverse transcription to synthesize 
Table I. siRNA duplexes and specificity used in study.

\begin{tabular}{llcc}
\hline siRNA & \multicolumn{1}{c}{ Sense strand } & Antisense strand & Specificity \\
\hline Control siRNA & UUCUCCGAACGUGUCACGUTT & ACGUGACACGUUCGGAGAATT & None \\
TGF $\beta 2$-192-siRNA & CGGAGGUGAUUUCCAUCUATT & CGGAGGUGAUUUCCAUCUAT & Rat \\
TGFß2-531-siRNA & GGGUCUUUCGCUUGCAGAATT & UUCUGCAAGCGAAAGACCCTG & Rat \\
TGFß2-771-siRNA & CCUUCAUACCGUCUAAUAATT & UUAUUAGACGGUAUGAAGGTA & Rat \\
\hline
\end{tabular}

Table II. Oligonucleotide primers used in study.

\begin{tabular}{lll}
\hline Primer & \multicolumn{1}{c}{ Sense strand } & \multicolumn{1}{c}{ Antisense strand } \\
\hline GAPDH & GCAAGTTCAACGGCACAG & GCCAGTAGACTCCACGACAT \\
TGF 2 & TGCCATCCCGCCCACTTTCTAC & CAATCCGTTGTTCAGCCACTCT \\
\hline
\end{tabular}

A

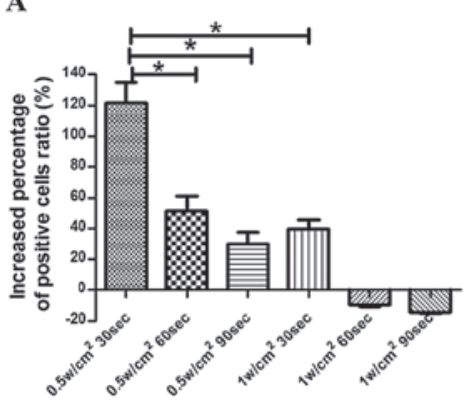

D

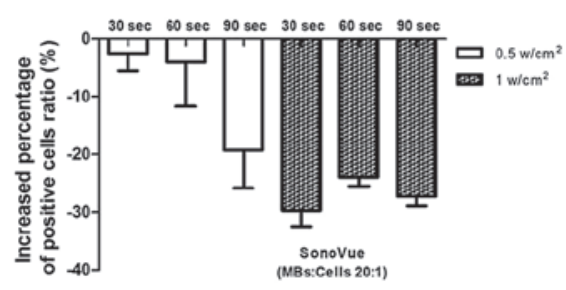

B

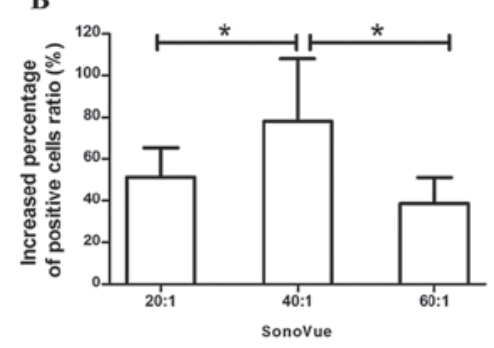

$\mathbf{E}$

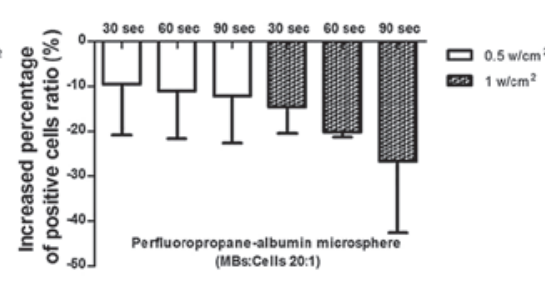

C
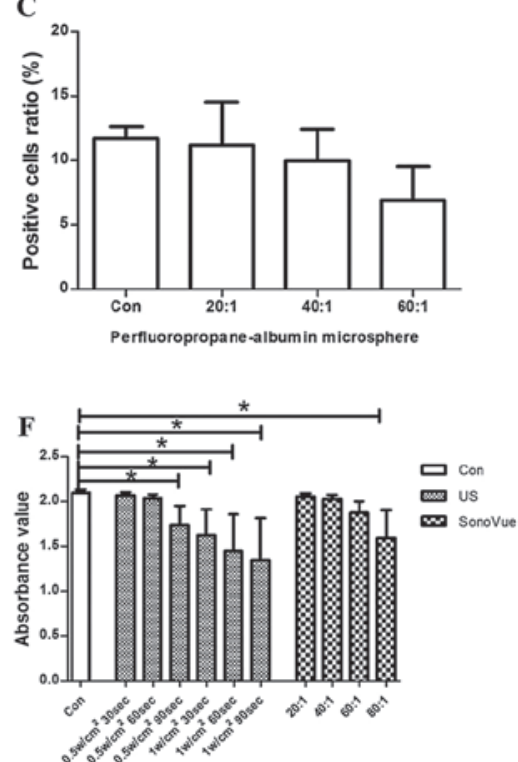

Figure 1. Effect of ultrasound (US) intensity, exposure time and microbubbles (MB) on type 2 recombinant adeno-associated virus transfection efficiency in rat retinal pigment epithelium cells. (A) Under conditions of $0.5 \mathrm{~W} / \mathrm{cm}^{2}$ and $30 \mathrm{sec}$, the increased ratio of positive cells was significantly higher than that of other groups. (B) Under the condition of a MB:cell ratio of 40:1, the increased ratio of positive cells was significantly higher than that of the 20:1 and 60:1 groups. (C) There was no difference between groups in which cells were treated with MBs and the control group. (D and E) Transfection efficiency of the US+SonoVue and US+perfluoropropane-albumin microsphere groups was decreased. (F) Absorbance values under the conditions of $0.5 \mathrm{~W} / \mathrm{cm}^{2}, 90 \mathrm{sec} ; 1 \mathrm{~W} / \mathrm{cm}^{2}, 30,60$ and $90 \mathrm{sec}$ and SonoVue MB:cell ratio of 80:1 were significantly lower than those of the control group. ${ }^{*} \mathrm{P}<0.05$.

cDNA was achieved using a reverse transcription system (Promega). RT-qPCR was performed with the extracted total RNA using Taq PCR Master mix (Toyobo Co., Ltd., Osaka, Japan). Quantification using the $2^{-\Delta \Delta C T}$ analytical method was performed in triplicate with GAPDH used for internal standardization. Oligonucleotide primers used for the analysis of gene expression were purchased from Sangon Co. Ltd. (Shanghai, China), as listed in Table II.

Statistical analysis. All values are expressed as the means \pm standard deviation. All cell viability experiments were performed in 12 parallel wells. All transfection experiments were performed in three parallel wells. An identical procedure was followed and repeated three times. One way analysis of variance was used to determine the significance of the differences in multiple comparisons. $\mathrm{P}<0.05$ was considered to indicate a statistically significant difference. The software package used was GraphPad Prism, version 5.0 (GraphPad Software, Inc, La Jolla, CA, USA).

\section{Results}

rAAV-based transfection in RPE-J cells with US and/or contrast agent.

Gene transfer by US. The US intensities and exposure times were set at 0.5 and $1 \mathrm{~W} / \mathrm{cm}^{2}$, and at 30,60 and $90 \mathrm{sec}$, respec- 

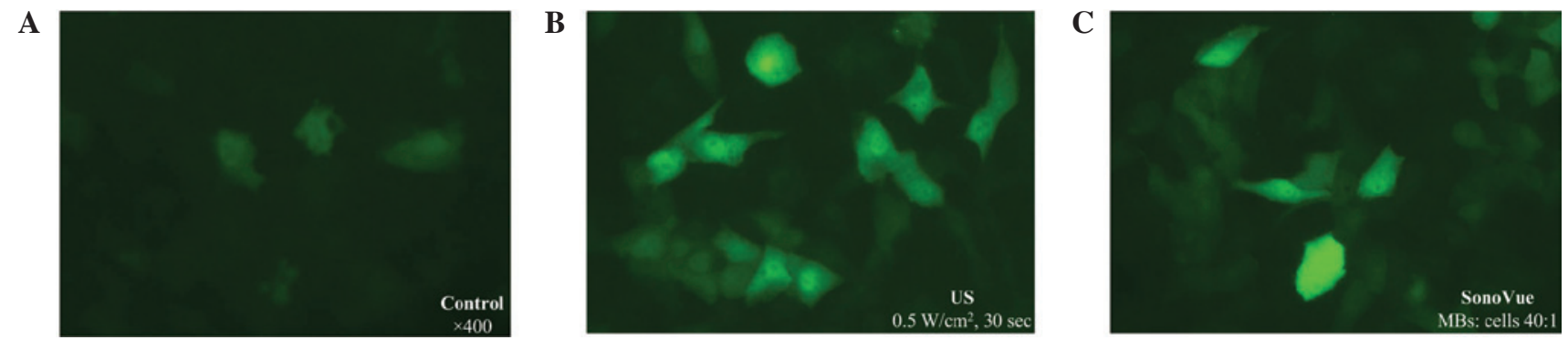

Figure 2. Observation of optimal ultrasound and SonoVue conditions on type 2 recombinant adeno-associated virus transfection efficiency in rat retinal pigment epithelium cells under a fluorescence microscope. Magnification, $\mathrm{x} 400$. The ratio and fluorescence intensity of positive cells in the ultrasound (B) and SonoVue (C) groups were higher than in the control group (A).
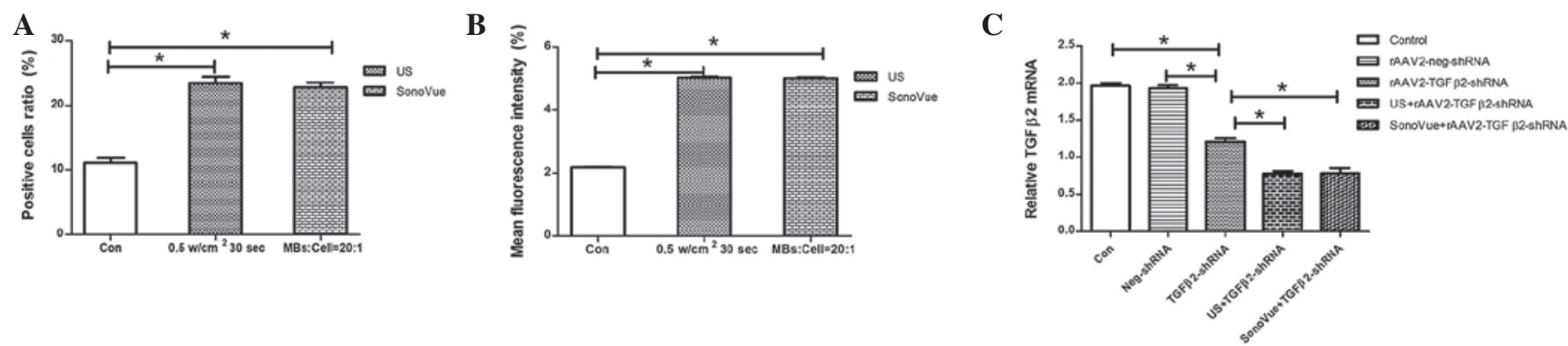

Figure 3. Transfection efficiency and TGF $\beta 2$ mRNA levels after rAAV2-TGF $\beta 2$ shRNA-EGFP was combined with ultrasound (US) or SonoVue. Under optimal conditions, US $\left(0.5 \mathrm{w} / \mathrm{cm}^{2}\right.$ and $\left.30 \mathrm{sec}\right)$ and microbubbles (MB:cell ratio, 40:1) significantly enhanced the rAAV2-TGF $\beta 2$ shRNA-EGFP positive cell ratio (A) and mean fluorescence density (B) compared with the control group. (C) Relative TGF $\beta 2 \mathrm{mRNA}$ levels in RPE-J cells $48 \mathrm{~h}$ after transfection with rAAV2-TGF $\beta 2$ shRNA-EGFP alone or combined with US or SonoVue. TGF $\beta 2$ transforming growth factor- $\beta 2$; rAAV2, type 2 recombinant adeno-associated virus; EGFP, enhanced green fluorescent protein. ${ }^{\text {}} \mathrm{P}<0.05$.

tively ( $50 \%$ duty cycle). Under the conditions of $0.5 \mathrm{~W} / \mathrm{cm}^{2}$ and $30 \mathrm{sec}$, the increased ratio of positive cells was significantly higher than that of other groups $(\mathrm{P}<0.0001$; Figs. 1A, 2A and $2 \mathrm{~B})$. With US intensity of $1 \mathrm{~W} / \mathrm{cm}^{2}$ and exposure time of 60 and $90 \mathrm{sec}$, transfection efficiency decreased rather than increased (Fig. 1A).

Gene transfer by SonoVue. The ratios of MBs to cells that we selected for our study were 20:1, 40:1 and 60:1. With the ratio of 40:1, the increased ratio of positive cells was significantly higher than that observed in the 20:1 and 60:1 groups ( $\mathrm{P}=0.011$ and $\mathrm{P}<0.0001$; Figs. 1B, 2A and 2C).

Gene transfer by perfluoropropane-albumin microspheres. The ratios of MBs to cells were 20:1, 40:1 and 60:1. There was no difference between those groups in which cells were treated with MBs and the control group ( $\mathrm{P}>0.05$, Fig. 1C).

Gene transfer by US/SonoVue or US/perfluoropropanealbumin microspheres. With various combinations of the parameters (intensity 0.5 and $1 \mathrm{~W} / \mathrm{cm}^{2}$; exposure time 30,60 and $90 \mathrm{sec}$; MB:cell ratio, 20:1) the ratios of positive cells in the UTMD groups showed a decrease instead of an increase (Fig. 1D and E).

\section{Cell viability assay}

US intensity and exposure duration. The US intensity was 0.5 or $1 \mathrm{~W} / \mathrm{cm}^{2}$, duration was 30,60 or $90 \mathrm{sec}$ and the duty cycle was $50 \%$. Cell viability decreased in sequence. Absorbance values under the conditions of $0.5 \mathrm{~W} / \mathrm{cm}^{2}$ and $90 \mathrm{sec}$, and
$1 \mathrm{~W} / \mathrm{cm}^{2}$ and 30,60 or $90 \mathrm{sec}$ were significantly lower than those of the control group $(\mathrm{P}=0.003, \mathrm{P}<0.0001, \mathrm{P}<0.0001$ and $\mathrm{P}<0.0001$, respectively; Fig. 1F).

Dosage of SonoVue. An MB:cell ratio of 80:1 was capable of significantly damaging cells compared with the control group $(\mathrm{P}<0.0001$; Fig. 1F). There was no difference between ratios of 20:1, 40:1 and 60:1 and the control group ( $\mathrm{P}>0.05)$.

rAAV2-TGFB2 shRNA-EGFP-based transfection in RPE-J cells with US or SonoVue and TGF 22 gene silencing evaluation. Under optimal conditions, both US $\left(0.5 \mathrm{~W} / \mathrm{cm}^{2}\right.$ and $30 \mathrm{sec}$ ) and SonoVue (MB:cell ratio, 40:1) significantly enhanced the rAAV2-TGF 32 shRNA-EGFP ratio of positive cells $(\mathrm{P}<0.0001$ and $\mathrm{P}<0.0001$; Fig. $3 \mathrm{~A})$ and mean fluorescence density $(\mathrm{P}<0.0001$ and $\mathrm{P}<0.0001$; Fig. $3 \mathrm{~B})$ compared with those of the control group. As shown in Fig. 3C, $48 \mathrm{~h}$ after transfection, the relative TGF $\beta 2$ mRNA level in RPE-J cells of rAAV2-TGF 32 shRNA-EGFP was markedly reduced compared with that of the control group and rAAV2-neg shRNA-EGFP group $(\mathrm{P}<0.0001$ and $\mathrm{P}<0.0001)$. TGF 32 gene silencing with a combination of US and rAAV2-TGF 32 shRNA-EGFP resulted in a significant decrease in the mRNA expression level compared with the rAAV2-TGF $\beta 2$ shRNA-EGFP alone group $(\mathrm{P}<0.0001)$. TGF $\beta 2$ gene silencing with a combination of SonoVue and rAAV2-TGF $\beta 2$ shRNA-EGFP also resulted in a significant decrease in the mRNA expression level $(\mathrm{P}<0.0001)$. No significant difference was observed between the control group and the rAAV2-neg-shRNA-EGFP group ( $\mathrm{P}>0.05)$. In addition, no 
significant difference was observed between the US group and the SonoVue group $(\mathrm{P}>0.05)$.

\section{Discussion}

The abnormal proliferation and migration of RPE cells play a crucial role in the onset and development of PVR (1). Several studies have demonstrated that the inhibition of TGF- $\beta$ has inhibitory effects on the proliferation of RPE, the formation of ECM and collagen contraction, and reduces the severity of PVR $(11,12)$. These studies also suggest that targeting of the TGF 32 gene with a siRNA in RPE cells has the potential to prevent the development of PVR.

Researchers have considered RPE cells to be a difficult cell type to transfect $(13,14)$. Adeno-associated virus (AAV) vectors have a number of significant advantages over other vectors, in particular the ability to induce long-term transgene expression in the eye and a relative lack of pathogenicity. The identification of the AAV vector of RNAi provides the premise for enhanced use of RNAi technology to silence related genes in the treatment of ophthalmic diseases.

However, the transduction of AAV occurs with relatively low efficiency, which limits its therapeutic effects. Several methods to increase the transfection efficiency of AAV vectors have been explored, including combinational use with an adenovirus or a chemotherapy drug $(7,8)$. However, the above strategies have a number of limitations. A new and improved method of delivery is required to improve AAV infection with enhanced performance in RPE cells.

In 1987, Fechheimer et al firstly proposed that US was able to promote gene transfection in vitro (15). The mechanisms of US-mediated transfection or UTMD enhancing gene transfection are not clear. We performed a series of studies on UTMD enhancing gene transfection in RPE cells (data not shown), including various vectors (rAAV, plasmid, PEI, liposome), various genes (pDNA, siRNA) and various cell lines (RPE-J and ARPE19 cells). Moreover, we proved that UTMD could be used safely to enhance and accelerate rAAV2-EGFP transgene expression of the retina (9). The goal of this feasibility study was to assess whether US and/or MBs could not only facilitate the delivery of rAAV2-EGFP but also downregulate TGF 32 combined with rAAV2-TGF 32 shRNA in rat RPE cells.

First, we observed the efficiency (ratio of positive cells) of UTMD combined with rAAV in driving transcription in RPE-J cells. The results reveal that US increased rAAV transfection efficiency. Under the conditions of $0.5 \mathrm{~W} / \mathrm{cm}^{2}$ and $30 \mathrm{sec}$, the increased transfection efficiency was significantly higher than that of other groups. Moreover, with an increase in US intensity and exposure time, the transfection efficiency and cell survival rate decreased. A 'window' of US intensity and exposure time exists and affects gene transfer efficiency. Higher or lower strength and longer or shorter exposure are not conducive to reaching the peak of gene transfer efficiency. With an increase in US intensity, the production probability of acoustic induced pores increased. With a long exposure time, the biological effect of cells increased, thereby affecting the cell survival rate. Under the conditions of $0.5 \mathrm{~W} / \mathrm{cm}^{2}$ and $30 \mathrm{sec}$, the energy was sufficient for the formation of transient pores in RPE-J cells and safe for cells.
The study demonstrated that rAAV combined with perfluoropropane-albumin microspheres without US was unable to increase rAAV transfection efficiency in RPE-J cells; however, SonoVue was able to do this. The differences in the two types of contrast agent were as follows: i) different composition of the membranes: lipid-shelled vs. albumin-shelled; ii) different components of wrapping gases: sulfur hexafluoride gas vs. perfluoropropane gas; iii) different size of microbubbles: 2.5-6.0 mm vs. 3-4.5 $\mu \mathrm{m}$. We also used two types of MBs combined with rAAV-transfected ARPE19 cells, and neither SonoVue nor perfluoropropane-albumin microspheres had a positive effect (data not shown). RPE-J cells produced effective endocytosis of phospholipid membranes using SonoVue. There are few studies on the effect of different types of MBs on gene transfection efficiency. One study, comparing the effect of different microbubble contrast agents on gene transfection, demonstrated that Optison improved pDNA transfection efficiency in the skeletal muscle of mice, but SonoVue and Levovist did not (16). The nonspecific phagocytic ability of cells may be the key factor in this notable phenomenon.

UTMD did not increase rAAV transfer to RPE-J cells but led to higher cell damage. MBs acting as cavitation nuclei effectively focus US energy and further potentiate bioeffects. Under UTMD conditions, the energy became too much for RPE-J cells and led to the high death rate of cells.

Whether US or MBs could damage RPE-J cells was also evaluated. With the increase in US intensity and exposure time, the survival rate of cells declined. High energy could lead to cell membrane fatigue, so that the corresponding position of the cell membrane damaged. Researchers have put most focus on whether US exposure or US combined with MBs affected the survival rate of cells. Few of them have paid attention to whether the US contrast agent itself caused cell damage. In our study, with the increase in MBs, the survival rate of cells declined. It is possible that the increase in MB concentration affected the living environment of the cell; for example, the osmotic pressure.

The combination of UTMD and rAAV should involve no destruction to the rAAV activity. Our previous study reveals that, under the UTMD conditions of intensity $\leq 3 \mathrm{~W} / \mathrm{cm}^{2}$, exposure time $\leq 120 \mathrm{sec}, \mathrm{MB}$ :cell ratio $\leq 50: 1$, frequency $1 \mathrm{MHz}$, $50 \%$ duty cycle and pulse repetition frequency of $100 \mathrm{~Hz}$, UTMD had no apparent effect on the transfection ability of rAAV (9). Therefore, the parameters used in this study were safe for rAAV and cells.

The preconditions for the realization of RNAi are safe and efficient importation into the target cells, as well as long-term stable effect. The first part of our study proved that US or SonoVue facilitates rAAV delivery to RPE-J cells. The second part of the study aimed to prove that US or SonoVue could facilitate rAAV-TGF $\beta 2$ shRNA-EGFP delivery to RPE-J cells and downregulate the expression of TGF $\beta 2$ mRNA efficiently. The efficiency (ratio of positive cells and mean fluorescence density) of US or SonoVue combined with rAAV-TGF $\beta 2$ shRNA-EGFP was observed in driving transcription in RPE cells. US and SonoVue enhanced rAAV transfection efficiency by increasing the transgene expression per cell (an increase of 111.8 and $105.8 \%$, respectively) and the percentage of transfected cells (an increase of 131.2 and $129.8 \%$, respectively). Then RT-qPCR was performed to evaluate the relative TGF 
mRNA levels in the groups. It was observed that the enhanced TGF $\beta 2$ gene silencing under the condition of rAAV-TGF 32 shRNA-EGFP combined with US or SonoVue resulted in a significant decrease in mRNA expression levels compared with rAAV-TGF 32 shRNA treatment alone.

The mechanism by which rAAV delivery efficiency is enhanced by US and MBs is controversial $(17,18)$. Only two public papers have been identified from PubMed research on this topic. One study suggested endocytosis as a mechanism that contributed to UTMD-enhanced AAV delivery (18). The other indicated that US enabled direct entry of the AAV vectors into the cytoplasm (17). In our study, SonoVue alone also enhanced AAV transfection and assisted in gene silencing. Although a study exists on the phenomenon (16), there are no studies on the mechanism by which MBs alone enhance gene transfection efficiency. The mechanism by which rAAV delivery efficiency is enhanced in RPE-J cells by US or SonoVue is worthy of additional study.

In the present study, we demonstrated that low-intensity US or an appropriate dose of SonoVue could be used safely to increase the delivery efficiency of rAAV-TGF 32 shRNA-EGFP to RPE-J cells. A combination of the biological (rAAV-TGF 32 shRNA-EGFP) and physical (US) approaches could more effectively downregulate the mRNA expression of TGF $\beta 2$ than rAAV alone. RPE cells in vivo were pentagonal or hexagonal, and the morphology of RPE cells cultured in vitro was mainly spindle. When the cells were cultured in vitro, they lost neurohumoral regulation and the influence of adjacent cells, living in an environment with an absence of dynamic balance. Thus, the morphology and function would change to a certain extent. Further studies are required to evaluate whether the delivery of rAAV-TGF 32 shRNA to RPE cells in a rat model of PVR may be enhanced by US and/or SonoVue.

\section{Acknowledgements}

This study was supported by the National Natural Science Foundation of China (81200700) and the Natural Science Foundation of Shanghai (11ZR1421100).

\section{References}

1. Chen Z, Chen CZ, Gong WR, Li JP and Xing YQ: Integrin-alpha5 mediates epidermal growth factor-induced retinal pigment epithelial cell proliferation and migration. Pathobiology 77: 88-95, 2010.

2. Carrington L, McLeod D and Boulton M: IL-10 and antibodies to TGF- $\beta 2$ and PDGF inhibit RPE-mediated retinal contraction. Invest Ophthalmol Vis Sci 41: 1210-1216, 2000.
3. Sugioka K, Kodama A, Okada K, et al: TGF- $\beta 2$ promotes RPE cell invasion into a collagen gel by mediating urokinase-type plasminogen activator (uPA) expression. Exp Eye Res 115: 13-21, 2013.

4. Lee SY, Huh MS, Lee S, et al: Stability and cellular uptake of polymerized siRNA (poly siRNA)/polyethylenimine (PEI) complexes for efficient gene silencing. J Control Release 141: 339-346, 2010.

5. Li Q, Dinculescu A, Shan Z, et al: Downregulation of p22phox in retinal pigment epithelial cells inhibits choroidal neovascularization in mice. Mol Ther 16: 1688-1694, 2008.

6. Askou AL, Pournaras JA, Pihlmann M, et al: Reduction of choroidal neovascularization in mice by adeno-associated virus-delivered anti-vascular endothelial growth factor short hairpin RNA. J Gene Med 14: 632-641, 2012.

7. Zhang SH, Wu JH, Wu XB, et al: Distinctive gene transduction efficiencies of commonly used viral vectors in the retina. Curr Eye Res 33: 81-90, 2008.

8. Wu JH, Zhang SH, Wu XB, et al: Enhanced transduction and improved photoreceptor survival of retinal degeneration by the combinatorial use of rAAV2 with a lower dose of adenovirus. Vision Res 48: 1648-1654, 2008.

9. Li HL, Zheng XZ, Wang HP, Li F, Wu Y and Du LF: Ultrasound-targeted microbubble destruction enhances AAV-mediated gene transfection in human RPE cells in vitro and rat retina in vivo. Gene Ther 16: 1146-1153, 2009.

10. Zheng XZ, Wu Y, Li HL, Du LF, Wang HO and Gu Q: Comparative analysis of the effects of ultrasound-targeted microbubble destruction on recombinant adeno-associated virus-and plasmid-mediated transgene expression in human retinal pigment epithelium cells. Mol Med Rep 2: 937-942, 2009.

11. Gamulescu MA, Chen Y, He S, et al: Transforming growth factor beta2-induced myofibroblastic differentiation of human retinal pigment epithelial cells: regulation by extracellular matrix proteins and hepatocyte growth factor. Exp Eye Res 83: 212-222, 2006.

12. Oshima Y, Sakamoto T, Hisatomi T, et al: Gene transfer of soluble TGF-beta type II receptor inhibits experimental proliferative vitreoretinopathy. Gene Ther 9: 1214-1220, 2002.

13. Sunshine JC, Sunshine SB, Bhutto I, Handa JT and Green JJ: Poly ( $\beta$-amino ester)-nanoparticle mediated transfection of retinal pigment epithelial cells in vitro and in vivo. PLoS One 7: e37543, 2012.

14. del Pozo-Rodriguez A, Delgado D, Solinis MA, Gascon AR and Pedraz JL: Solid lipid nanoparticles for retinal gene therapy: transfection and intracellular trafficking in RPE cells. Int $\mathbf{J}$ Pharm 360: 177-183, 2008.

15. Fechheimer M, Boylan JF, Parker S, Sisken JE, Patel GL and Zimmer SG: Transfection of mammalian cells With plasmid DNA by scrape loading and sonication loading. Proc Natl Acad Sci 84: 8463-8467, 1987.

16. Wang X, Liang HD, Dong B, Lu QL and Blomley MJ: Gene transfer with microbubble ultrasound and plasmid DNA into skeletal muscle of mice: comparison between commercially available microbubble contrast agents. Radiology 237: 224-249, 2005.

17. Geers B, Lentacker I, Alonso A, et al: Elucidating the mechanisms behind sonoporation with adeno-associated virus-loaded microbubbles. Mol Pharm 8: 2244-2251, 2011.

18. Jin LF, Li F, Wang HP, Wei F, Qin P and Du LF: Ultrasound targeted microbubble destruction stimulates cellular endocytosis in facilitation of adeno-associated virus delivery. Int $\mathbf{J}$ Mol Sci 14: 9737-9750, 2013. 